\title{
Prenatal diagnosis and management of a fetal neck mass
}

\author{
Emek Doğer, Yasin Ceylan, Ahmet Yiğit Çakıroğlu, Eray Çalsşkan \\ Department of Obstetrics and Gynecology, Kocaeli University Faculty of Medicine, Kocaeli, Turkey
}

\begin{abstract}
We report the case of a benign mesenchymal spindle-cell tumor located on fetal neck, diagnosed during prenatal ultrasound and magnetic resonance investigation. A 30-year-old woman (gravida 2, para 1) was referred to our perinatology unit for evaluation of a fetal neck mass that had been identified on ultrasonography at 29 weeks gestation. A right lateral neck mass was observed (size: $42 \times 40 \mathrm{~mm}$ ) that extended from the preauricular region to right clavicula. Fetal MRI revealed a solid heterogeneous mass arising from the right lateral neck and there was no invasion around tissue and no extension of the mass into the chest. At 37 weeks after birth, we observed that the mass was subcutaneous and there was no invasion to the surrounding tissue. The trachea was not compressed and there was no extension of the mass into the chest. Then, the neck mass was completely resected after birth without any complications. Histopathological examination of tumor was consistent with mesenchymal spindle-cell tumor. Immunohistochemical staining with CD34 and actin was positive; however, caldesmone, epithelial membrane antigen (EMA), and S-100 was negative. Fetal MRI performed during the pregnancy for investigation of fetal neck masses detected on ultrasound gives compatible results observed in the neonate after birth and maintains adequate findings for follow-up and planning of treatment. ( $\mathrm{J}$ Turk Ger Gynecol Assoc 2015; 16: 118-20)
\end{abstract}

Keywords: Fetal neck mass, fetal magnetic resonance imaging, mesenchymal spindle-cell tumor

Received: 29 August, 2013

Accepted: 17, October, 2013

\section{Introduction}

Congenital tumors are extremely uncommon in infants, particularly in the head and neck region (1). The most common fetal neck mass is cystic higroma; cervical teratoma is the most common the fetal neck tumor. Fetal neck masses are uncommon and may not be apparent during the second trimester ultrasonography (2). Differential diagnosis of fetal neck tumors is difficult to be detected by ultrasonography. Encephalomyelocele, lymphangioma/higroma, teratoma, sarcoma, haemangioma, neuroblastoma, and goiter should be included in the differential diagnosis of fetal neck masses (3). Prenatal diagnosis of fetal neck mass has improved the survival and morbidity of infants with giant neck masses (4). Prenatal ultrasonography and MRI may enhance the accuracy of antenatal diagnosis (location, extension, and intracranial spread) and help in the selection of patients who require treatment (5). In this case report, we present a solid heterogeneous mass arising from the right lateral neck with radiological, histological, and immunohistochemical findings.

\section{Case Presentation}

A 30 -year-old woman (gravida 2, para 1) was referred to our perinatology unit for the evaluation of a fetal neck mass that had been identified on ultrasonography at 29 weeks gestation. A right lateral neck mass (size: $42 \times 40 \mathrm{~mm}$ ) was observed, extending from the preauricular region to right clavicle. Generally, the mass had a solid component (Figure 1). We obtained consent for performing a fetal MRI from the family. The solid heterogeneous mass arising from the right lateral neck, without signs of invasion to surrounding tissues and no extension into the chest in three planes MRI images (Figure 2). At 37 weeks, C-section was performed due to the early membrane rupture. After parental consent was obtained for pictures, the macroscopic appearance of the newborn is shown in Figure 3. The Apgar scores were 8 and 9 at 1 and $5 \mathrm{~min}$, respectively. The infant was a male who weighed $3150 \mathrm{~g}$ at birth. After birth, we obtained consent from the family for MRI in three planes, confirming the presence of a solid heterogeneous mass arising from the right lateral neck (Figure 4). The mass was subcutaneous and there was no invasion of surrounding tissues. The trachea was not compressed. There was no extension into the chest. Then, the neck mass was completely resected after the birth without complication. Histopathological examination of the tumor was consistent with mesenchymal spindle-cell tumor. Immunohistochemical staining with CD34 and actin was positive; however, caldesmone, epithelial membrane antigen (EMA), and S-100 were negative. Morphological examination confirmed the diagnosis of myofibroma or infantile hemangioperistoma. 


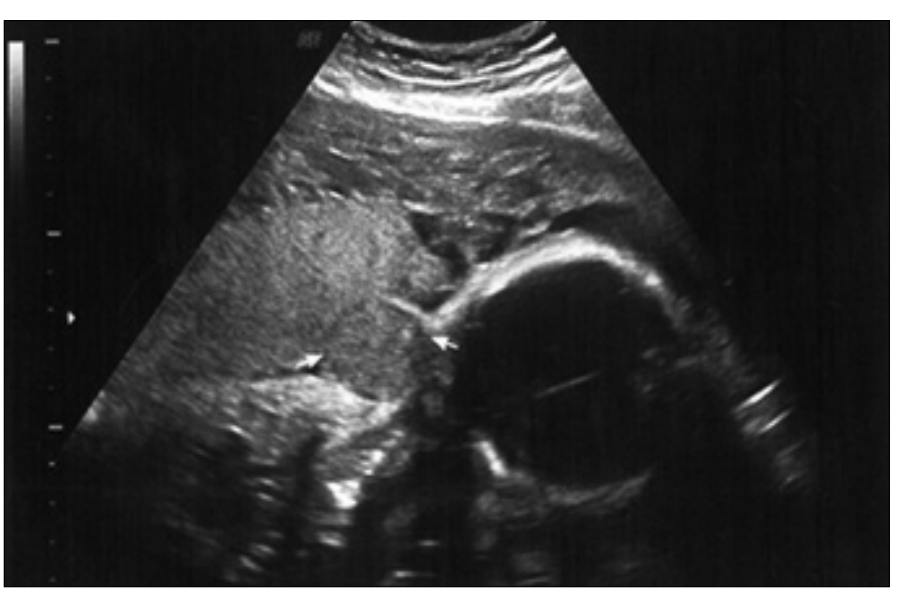

Figure 1. Sonographic image at 28 weeks and six days

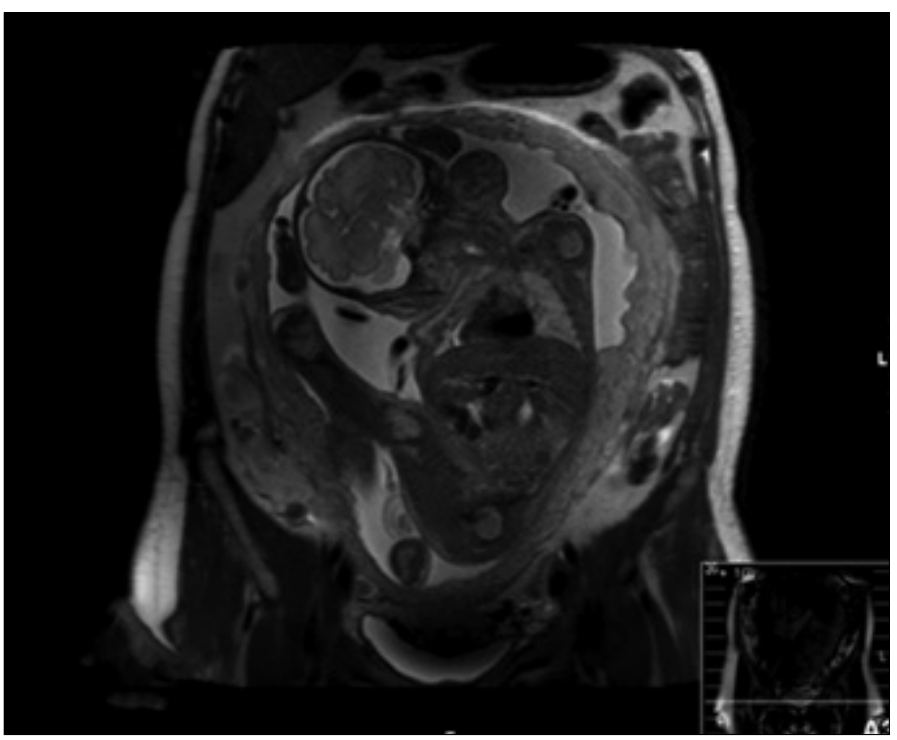

Figure 2. Fetal MRI image at 30 weeks and five days

\section{Discussion}

Congenital cervical tumors can be subdivided into anterior and posterior masses (2). In the anterior neck area, other masses can be caused by soft-tissue lesions, such as hamartomas, sarcomas, or goiter, and they may cause hyperextension of the neck (3). Large masses can have major fetal and perinatal effects due to the compression and distortion of surrounding cervical structures. Compression from a large lesion on the fetal esophagus and trachea can cause impaired fetal swallowing, polyhydramnios, and preterm labor in the prenatal period, and airway obstruction, hypoxia, and death after delivery $(4,6)$. After diagnosis, multidisciplinary prenatal management, including nondirective counseling, serial imaging, and planned delivery, was incorporated (6). Fetal ultrasonography helps to visualize the vascularity and consistency of the mass (solid or cystic) and can determine indirect signs of esophageal or tracheal obstruction. Fetal MRI enhances sensitivity in characterizing the extent of lesion infiltration and distorted anatomy of the neck structures. MRI is also useful in further delineating

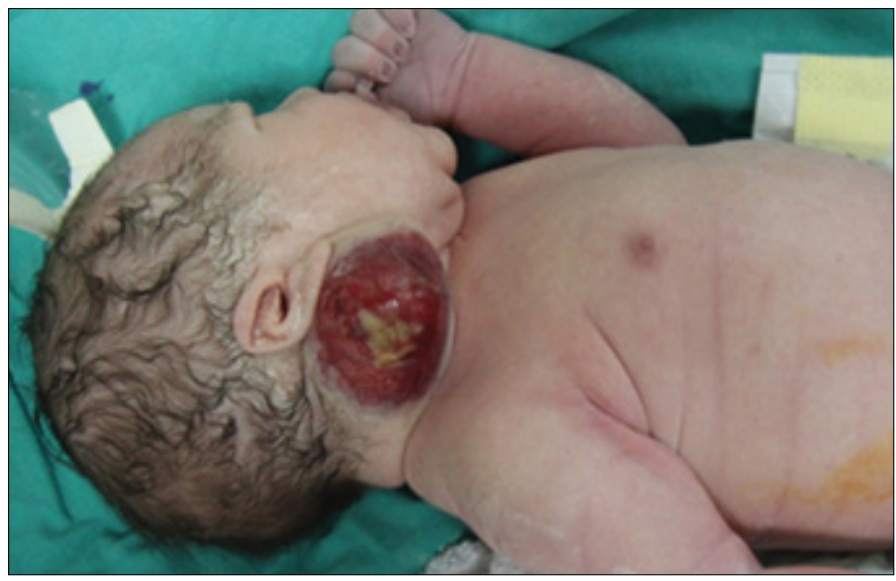

Figure 3. Macroscopic appearance after the c-section

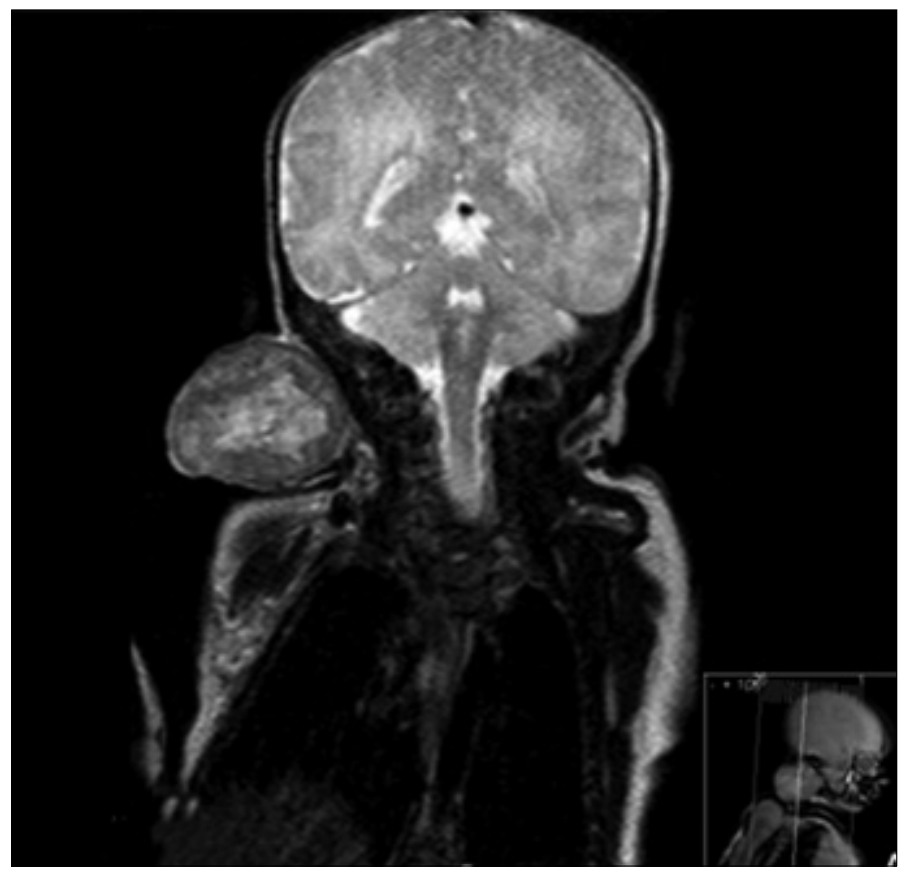

Figure 4. MRI image of infant after birth

lesions of neural and vascular origin (4). Antenatal fetal MRI and ultrasonography therefore it may be critical in identifying fetal neck masses that require ex utero intrapartum treatment (EXIT) procedure (5). Consequently, it is widely accepted that the cell of origin of all soft-tissue sarcomas is a primitive mesenchymal cell that can differentiate in many different directions. Fibromatoses, fibrosarcomas, neurofibrosarcomas, leiomyosarcomas, rhabdomyosarcomas, liposarcomas, angiosarcomas, mesotheliomas, and meningiomas are soft tissue sarcomas (7). Clinically, four common principles apply to spindle-cell sarcomas and soft-tissue sarcomas: the more superficial the location, the more likely the tumor is to be benign; if the tumor location is superficial, it is generally benign (deep tumors tend to be malignant); the larger the tumor, the more chances that it is malignant; and a rapidly growing tumor is generally more likely to be malignant. Benign tumors are relatively avascular, however most malignancies are hypervascular $(7,8)$. The most 
common treatment of choice is excision; extensive excision or amputation should be performed when anatomically possible because spindle-cell sarcomas often infiltrate deep fascial planes that make it difficult to identify on gross examination of peripheral margins of the tumor. Those sarcomas have a greater potential for metastasis, and the time between recurrences is usually short. Generally, spindle-cell sarcomas do not respond well to radiotherapy; however, higher doses have been reported to control approximately $50 \%$ of them for up to a year. Surgical resection followed by radiation is also an option for local control. Chemotherapy for sarcomas has become a more acceptable treatment modality. The chemotherapeutic protocols involve the use of adriamycin often in combination with other agents, including cyclophosphamide, vincristine, dacarbazine, and methotrexate (8).

Ethics Commitee Approval: Ethics committee approval was received for this study from the ethics committee of Local Instutional Ethics Committee.

Informed Consent: Written informed consent was obtained from patients who participated in this case.

Author Contributions: Concept - E.D.; Y.C.; Design - E.D.; Y.C.; Supervision - E.C.; Resource - Y.C.; Materials - Y.C.; Data Collection \& /or Processing - Y.C.; Analysis \& /or Interpretation - E.D.; Literature Search - Y.C.; Writing - E.D., Y.C.; Critical Reviews - E.C.

Conflict of Interest: No conflict of interest was declared by the authors.
Financial Disclosure: The authors declared that this study has received no financial support.

\section{References}

1. Gupta A, Maddalozzo J, Win Htin T, Shah A, Chou PM. Spindle cell rhabdomyosarcoma of the tongue in an infant: a case report with emphasis on differential diagnosis of childhood spindle cell lesions. Pathol Res Pract 2004; 200: 537-43. [CrossRef]

2. Rauff S, Kien TE. Ultrasound diagnosis of fetal neck masses: a case series. Case Rep Obstet Gynecol 2013; 2013: 243590. [CrossRef]

3. Güzelmansur I, Aksoy HT, Hakverdi S, Seven M, Dilmen U, Dilmen G. Fetal cervical neuroblastoma: prenatal diagnosis. Case Rep Med 2011; 2011: 529749. [CrossRef]

4. Cass DL. Impact of prenatal diagnosis and therapy on neonatal surgery. Semin Fetal Neonatal Med 2011; 16: 130-8. [CrossRef]

5. Tonni G, De Felice C, Centini G, Ginanneschi C. Cervical and oral teratoma in the fetus: a systematic review of etiology, pathology, diagnosis, treatment and prognosis. Arch Gynecol Obstet 2010; 282: 355-61. [CrossRef]

6. Lazar DA, Cassady CI, Olutoye OO, Moise KJ Jr, Johnson A, Lee TC, Cass DL. Tracheoesophageal displacement index and predictors of airway obstruction for fetuses with neck masses. J Pediatr Surg 2012; 47: 46-50. [CrossRef]

7. Carew JF, Kraus DH. Clinical considerations for neoplasms of the neck. In: Fu Y-S, Wenig BM, Abemayor E, Wenig BL, editors. Head and Neck Pathology with Clinical Correlations. Philadelphia, PA: Churchill-Livingstone; 2001. p. 771-80.

8. Cynthia M. Kahn, Scott Line. Musculoskeletal system. The Merck Veterinary Manual, 10th Edition, 2010. 